doi:10.13108/2021-13-4-112

\title{
DUAL SPACES FOR WEIGHTED SPACES OF LOCALLY INTEGRABLE FUNCTIONS
}

\section{R.S. YULMUKHAMETOV}

Abstract. In this work we consider weighted $L_{2}$ spaces on convex domains in $\mathbb{R}^{n}$ and we study the problem on describing the dual space in terms of the Laplace-Fourier transform.

Let $D$ be a bounded convex domain in $\mathbb{R}^{n}$ and $\varphi$ be a convex function on this domain. By $L_{2}(D, \varphi)$ we denote the space of locally integrable functions $D$ with a finite norm

$$
\|f\|^{2}:=\int_{D}|f(t)|^{2} e^{-2 \varphi(t)} d t .
$$

Under some restrictions for the weight $\varphi$ we prove that an entire function $F$ is represented as the Fourier - Laplace transform of a function in $L_{2}(D, \varphi)$, that is,

$$
F(\lambda)=\int_{D} e^{t \lambda-2 \varphi(t) \overline{f(t)}} d t, \quad f \in L_{2}(D, \varphi),
$$

for some function $f \in L_{2}(D, \varphi)$ if and only if

$$
\|F\|^{2}:=\int \frac{|F(z)|^{2}}{K(z)} \operatorname{det} G(\widetilde{\varphi}, x) d y d x<\infty,
$$

where $G(\widetilde{\varphi}, x)$ is the Hessian matrix of the function $\widetilde{\varphi}$,

$$
K(\lambda):=\left\|\delta_{\lambda}\right\|^{2}, \quad \lambda \in \mathbb{C}^{n} .
$$

As an example we show that for the case, when $D$ is the unit circle and $\varphi(t)=(1-|t|)^{\alpha}$, the space of Fourier-Laplace transforms is isomorphic to the space of entire functions $F(z), z=x+i y \in \mathbb{C}^{2}$, for which

$$
\|F\|^{2}:=\int|F(x+i y)|^{2} e^{-2|x|-2(a \beta)^{\frac{1}{\beta+1}}(a+1)|x|^{\frac{\beta}{\beta+1}}}(1+|x|)^{\frac{\alpha-3}{2}} d x d y<\infty,
$$

where $\alpha=\frac{\beta}{\beta+1}$.

Keywords: weighted spaces, Fourier-Laplace transform, entire functions.

Mathematics Subject Classification: 32A15, 42B10

\section{INTRODUCTION}

Let $D$ be a bounded convex domain in $\mathbb{R}^{n}$ and $\varphi$ be a convex function on this domain. By $L_{2}(D, \varphi)$ we denote the space of locally integrable functions on $D$ with a finite norm

$$
\|f\|^{2}:=\int_{D}|f(t)|^{2} e^{-2 \varphi(t)} d t
$$

R.S. Yulmukhametov, Dual spaces to Weighted spaces of locally integrable functions.

(C) Yulmukhametov R.S. 2021.

The research is made in the framework of executing the Developing Program of Scientific and Educational mathematical center of Privolzhsky Federal District (agreement no. 075-02-2021-1393).

Submitted August 25, 2021. 
A system of functions $e^{t \lambda}$, where $t=\left(t_{1}, \ldots, t_{n}\right), \lambda=\left(\lambda_{1}, \ldots, \lambda_{n}\right) \in \mathbb{C}^{n}$ and $t \lambda=\sum_{k=1}^{n} t_{k} \lambda_{k}$, is complete in the Hilbert space $L_{2}(D, \varphi)$ and this is why the Fourier-Laplace transform

$$
\mathcal{L}: S \rightarrow S\left(e^{t \lambda}\right), \quad \lambda \in \mathbb{C}^{n},
$$

maps the dual space $L_{2}^{*}(D, \varphi)$ onto some space $\widehat{L}_{2}(D, \varphi)$ of the functions defined on $\mathbb{C}^{n}$. Since a Hilbert space coincides with its dual, the space $\widehat{L}_{2}(D, \varphi)$ consists of the functions of form

$$
\widehat{f}(\lambda)=\int_{D} e^{t \lambda-2 \varphi(t)} \overline{f(t)} d t, \quad f \in L_{2}(D, \varphi),
$$

in particular, $\widehat{L}_{2}(D, \varphi)$ is a subspace of the space of entire functions. The space $\widehat{L}_{2}(D, \varphi)$ is a Hilbert one with respect to the induced scalar product $(\widehat{f}, \widehat{g})=(f, g)$.

We note that the point functionals $\delta_{\lambda}: F \rightarrow F(\lambda)$ are continuous in the space $\widehat{L}_{2}(D, \varphi)$ for each $\lambda \in \mathbb{C}^{n}$. The function

$$
K(\lambda):=\left\|\delta_{\lambda}\right\|^{2}, \quad \lambda \in \mathbb{C}^{n},
$$

is called Bergman function.

In this paper we consider the issue on a weighted descriptions of the induced norm in this space. In the one-dimensional case this question was completely solved in work [2] and in a final formulation in work [1] the answer reads as follows.

Let $D$ be an interval in the real axis and $\varphi$ be a convex function on this interval. Then the space $\widehat{L}_{2}(D, \varphi)$ is isomorphic to the space of entire functions $F$ satisfying the conditions

$$
|F(z)| \leqslant C K(z), z \in \mathbb{C}, \quad\|F\|^{2}:=\int \frac{|F(z)|^{2}}{K(z)} d y d \widetilde{u}_{+}^{\prime}(x)<\infty .
$$

We shall assume that $\varphi \in C^{2}$ and that this function is strictly convex.

\section{Convex FunCtions}

In this section we introduce a regularity notion and expose some of its properties.

Let $K$ be a convex domain, $\psi \in C^{2}(K)$ be a strictly convex function and

$$
\nabla \psi(t)=\left(\frac{\partial \psi}{\partial t_{1}}(t), \ldots, \frac{\partial \psi}{\partial t_{n}}(t)\right)
$$

be a gradient vector, and

$$
G(\psi, t)=\left(\frac{\partial^{2} \psi}{\partial t_{i} \partial t_{j}}(t)\right)_{i, j=1}^{n}
$$

be the Hessian matrix of the function $\psi$ at a point $t \in K$.

The strict convexity of the function $\psi$ is equivalent to the positive definiteness of its Hessian matrix:

$$
(\omega, G(\psi, t) \omega)>0, \quad \omega \in \mathbb{R}^{n}, \quad\|\omega\|=1, \quad t \in D .
$$

In particular, the mapping $\nabla \psi(t)$ is injective for each $t \in K$. The function

$$
\widetilde{\psi}(\tau)=\sup _{t \in K}(t \tau-\psi(t))
$$

is called a Young transform. In the general case the Young transform $\widetilde{\psi}$ is a convex function in some convex domain $\widetilde{K}$. If the supremum is attained at an internal point of the domain $K$, then it follows from the inverse function theorem that the function $\widetilde{\psi}$ is differentiable at a point $\tau$ and $\nabla \widetilde{\psi}(\nabla \psi(\tau)) \equiv \tau$. Differentiating this identity, we see that the Hessian matrices satisfy the identity

$$
G(\widetilde{\psi}, \nabla \psi(t)) G(\psi, t)=E, \quad t \in D,
$$


where $E$ is the unit matrix. We shall the domain

$$
E\left(\psi, t_{0}, p\right)=\left\{t \in D:\left(t-t_{0}\right) G\left(\psi, t_{0}\right)\left(t-t_{0}\right)<p\right\}
$$

an $p$-ellipsoid of the function $\psi$ at a point $t_{0}$.

In the vicinity of each point $t_{0} \in K$ the function $\psi \in C^{2}$ is represented by the Taylor formula

$$
\psi(t)=\varphi\left(t_{0}\right)+\nabla \varphi\left(t_{0}\right)\left(t-t_{0}\right)+\frac{1}{2}\left(t-t_{0}\right) G\left(\varphi, t_{0}\right)\left(t-t_{0}\right)+\alpha\left(t_{0}, t-t_{0}\right)\left|t-t_{0}\right|^{2},
$$

where $\alpha\left(t_{0}, \varepsilon\right) \rightarrow 0$ as $\varepsilon \rightarrow 0$. For a positive $p$ we let

$$
\Omega\left(\psi, t_{0}, p\right)=\left\{t \in D: \psi(t)-\psi\left(t_{0}\right)-\nabla \psi\left(t_{0}\right)\left(t-t_{0}\right)<p\right\},
$$

then $\Omega\left(\psi, t_{0}, p\right)$ is some convex neighbourhood of the point $t_{0}$.

We introduce a condition: there exist numbers $q>1, p>0$ such that

$$
\begin{aligned}
\frac{1}{2 q}\left(t-t_{0}\right) G\left(\psi, t_{0}\right)\left(t-t_{0}\right) & \leqslant\left|\psi(t)-\psi\left(t_{0}\right)-\nabla \psi\left(t_{0}\right)\left(t-t_{0}\right)\right| \\
& \leqslant \frac{q}{2}\left(t-t_{0}\right) G\left(\psi, t_{0}\right)\left(t-t_{0}\right), \quad t \in E\left(\psi, t_{0}, p\right) .
\end{aligned}
$$

Lemma 2.1. Let $\varphi \in C^{2}$ be a strictly convex function in a bounded convex domain $D$ and $|\varphi(t)| \rightarrow+\infty$ as $\operatorname{dist}(t) \rightarrow 0$. If $\widetilde{\varphi}$ satisfies condition (2.1) at a point $x \in \mathbb{R}^{n}$, then

$$
E\left(\widetilde{\varphi}, x, \frac{p}{q}\right) \subset \Omega(\widetilde{\varphi}, x, p) \subset E\left(\widetilde{\varphi}, x, 4 q^{2} p\right)
$$

and

$$
c_{n}\left(\frac{p}{q}\right)^{\frac{n}{2}} \frac{1}{\sqrt{\operatorname{det} G(\widetilde{\varphi}, x)}} \leqslant|\Omega(\widetilde{\varphi}, x, p)| \leqslant c_{n} \frac{\left(4 p q^{2}\right)^{\frac{n}{2}}}{\sqrt{\operatorname{det} G(\widetilde{\varphi}, x)}},
$$

where the symbol $|A|$ stands for the volume of the set $A$, the symbol $\operatorname{dist}(t)$ denotes the distance from a point $t \in D$ to the boundary of $D$, while $c_{n}$ is the volume of the unit ball in $\mathbb{R}^{n}$.

Proof. Let us prove that $\Omega(\widetilde{\varphi}, x, p) \subset E\left(\widetilde{\varphi}, x, 4 q^{2} p\right)$. Without loss of generality we assume that $x=0$. We take $\tau^{\prime \prime} \in \partial \Omega(\widetilde{\varphi}, 0, p)$ :

$$
\widetilde{\varphi}\left(\tau^{\prime \prime}\right)-\widetilde{\varphi}(0)-\nabla \widetilde{\varphi}(0) \tau^{\prime \prime}=p
$$

If $\tau^{\prime \prime} \notin E(\widetilde{\varphi}, 0, p)$, then the segment connecting the point $\tau^{\prime \prime}$ with the point 0 intersects the boundary of the ellipsoid $E(\widetilde{\varphi}, 0, p)$ at some point $\tau^{\prime} \in \Omega(\widetilde{\varphi}, 0, p)$ :

$$
\tau^{\prime} G(\widetilde{\varphi}, 0) \tau^{\prime}=p .
$$

By condition (2.1),

$$
\widetilde{\varphi}\left(\tau^{\prime}\right)-\widetilde{\varphi}(0)-\nabla \widetilde{\varphi}(0) \tau^{\prime} \geqslant \frac{1}{2 q} \tau^{\prime} G(\widetilde{\varphi}, 0) \tau^{\prime}=\frac{p}{2 q} .
$$

The function $\widetilde{\varphi}(t)-\widetilde{\varphi}(0)-\nabla \widetilde{\varphi}(0) t$ is convex in $t$ and this is why

$$
p=\widetilde{\varphi}\left(\tau^{\prime \prime}\right)-\widetilde{\varphi}(0)-\nabla \widetilde{\varphi}(0) \tau^{\prime \prime} \geqslant \frac{\widetilde{\varphi}\left(\tau^{\prime}\right)-\widetilde{\varphi}(0)-\nabla \widetilde{\varphi}(0) \tau^{\prime}}{\left|\tau^{\prime}\right|}\left|\tau^{\prime \prime}\right| \geqslant \frac{p}{2 q} \frac{\left|\tau^{\prime \prime}\right|}{\left|\tau^{\prime}\right|} .
$$

Therefore, $\left|\tau^{\prime \prime}\right| \leqslant 2 q\left|\tau^{\prime}\right|$ and

$$
\tau^{\prime \prime} G(\widetilde{\varphi}, 0) \tau^{\prime \prime}=\frac{\left|\tau^{\prime \prime}\right|^{2}}{\left|\tau^{\prime}\right|^{2}} \tau^{\prime} G(\widetilde{\varphi}, 0) \tau^{\prime} \leqslant 4 q^{2} p,
$$

that is, $\tau^{\prime \prime} \in E\left(\widetilde{\varphi}, 0,4 q^{2} p\right)$ and this leads us to the needed inclusion.

Let us prove the inclusion $E\left(\widetilde{\varphi}, 0, \frac{p}{q}\right) \subset \Omega(\widetilde{\varphi}, 0, p)$. Let $\tau^{\prime \prime} \in \partial E\left(\widetilde{\varphi}, 0, \frac{p}{q}\right)$ :

$$
\tau^{\prime \prime} G(\widetilde{\varphi}, 0) \tau^{\prime \prime}=\frac{p}{q}
$$


and suppose that $\tau^{\prime \prime} \notin \Omega(\widetilde{\varphi}, 0, p)$ :

$$
\widetilde{\varphi}\left(\tau^{\prime \prime}\right)-\widetilde{\varphi}(0)-\nabla \widetilde{\varphi}(0) \tau^{\prime \prime}>p .
$$

The segment connecting the point $\tau^{\prime \prime}$ with the point 0 intersects the boundary of $\Omega(\widetilde{\varphi}, 0, p)$ at some point $\tau^{\prime}$ :

$$
\widetilde{\varphi}\left(\tau^{\prime}\right)-\widetilde{\varphi}(0)-\nabla \widetilde{\varphi}(0) \tau^{\prime}=p
$$

By condition (2.1),

$$
\tau^{\prime} G(\widetilde{\varphi}, 0) \tau^{\prime} \geqslant \frac{2}{q}\left(\widetilde{\varphi}\left(\tau^{\prime}\right)-\widetilde{\varphi}(0)-\nabla \widetilde{\varphi}(0) \tau^{\prime}\right)=\frac{2 p}{q}
$$

and therefore,

$$
\frac{p}{q}=\tau^{\prime \prime} G(\widetilde{\varphi}, 0) \tau^{\prime \prime}=\frac{\left|\tau^{\prime \prime}\right|^{2}}{\left|\tau^{\prime}\right|^{2}} \tau^{\prime} G(\widetilde{\varphi}, 0) \tau^{\prime} \geqslant \frac{\left|\tau^{\prime \prime}\right|^{2}}{\left|\tau^{\prime}\right|^{2}} \frac{2 p}{q},
$$

that is, $\left|\tau^{\prime \prime}\right|<\left|\tau^{\prime}\right|$ and $\tau^{\prime \prime} \in \Omega(\widetilde{\varphi}, 0, p)$. We have obtained a contradiction, which proves the inclusion $E\left(\widetilde{\varphi}, x, \frac{p}{q}\right) \subset \Omega(\widetilde{\varphi}, x, p)$.

The proven inclusions imply that

$$
\left|E\left(\widetilde{\varphi}, x, \frac{p}{q}\right)\right| \leqslant|\Omega(\widetilde{\varphi}, x, p)| \leqslant\left|E\left(\widetilde{\varphi}, x, 4 p q^{2}\right)\right| .
$$

If $A$ is a positive definite matrix, then the principal axes of the ellipse $x A x \leqslant p$ are equal to $\sqrt{\frac{p}{\lambda_{k}}}$, where $\lambda_{k}$ are the eigenvalues of the matrix $A$. The volume of the ellipse is equal to $c_{n} \frac{p^{\frac{n}{2}}}{\sqrt{\lambda_{1} \ldots \lambda_{n}}}$, where $c_{n}$ is the volume of the unit ball in $\mathbb{R}^{n}$ and $\operatorname{det} A=\lambda_{1} \ldots \lambda_{n}$. Thus,

$$
c_{n}\left(\frac{p}{q}\right)^{\frac{n}{2}} \frac{1}{\sqrt{\operatorname{det} G(\widetilde{\varphi}, x)}} \leqslant|\Omega(\widetilde{\varphi}, x, p)| \leqslant c_{n} \frac{\left(4 p q^{2}\right)^{\frac{n}{2}}}{\sqrt{\operatorname{det} G(\widetilde{\varphi}, x)}} .
$$

The proof is complete.

In work [1, the notion of the volume distance was introduced. It is defined by the induction in the dimension of the space as follows. Let $E$ be some convex domain in $\mathbb{R}^{n}, x \in E$. If $n=1$, then we let

$$
\operatorname{vd}(x, E)=\inf \{|x-y|: y \notin E\}
$$

the volume distance to be the usual distance from a point $x \in E$ to the boundary $E$. Suppose that the quantity $\operatorname{vd}(x, E)$ is defined in the space $\mathbb{R}^{n}$ and $E \subset \mathbb{R}^{n+1}$. We take a point $x_{0} \in \partial E$ such that

$$
\inf \{|x-y|: y \notin E\}=\left|x-x_{0}\right| \text {. }
$$

If the number of such points is greater than one, we take an arbitrary of them. The point $x_{0}$ is passed by a unique support hyperplane orthogonal to the segment connecting the points $x$ and $x_{0}$. Let $P$ be a hyperplane parallel to this support hyperplane and passing through the point $x$. The dimension of the convex set $E_{1}=P \cap E$ is equal to $n$ and $x \in E_{1}$. By the induction assumption, the quantity $\operatorname{vd}\left(x, E_{1}\right)$ is already defined. We let

$$
\operatorname{vd}(x, E)=\operatorname{vd}\left(x, E_{1}\right)\left|x-x_{0}\right| .
$$

For instance, for an ellipsoid $E$ in $\mathbb{R}^{n}$ with principal axes $a_{1}, \ldots, a_{n}$, which is centered at the origin, we see easily that $\operatorname{vd}(0, E)=a_{1} \ldots a_{n}$.

Lemma 2.2. Let $\varphi \in C^{2}$ be a strictly convex function in a bounded convex domain $D$ and $|\varphi(t)| \rightarrow+\infty$ as $\operatorname{dist}(t) \rightarrow 0$. If $\widetilde{\varphi}$ satisfies condition (2.1) at the point $x \in \mathbb{R}^{n}$, then

$$
\left(\frac{p}{2 q n}\right)^{n}(\operatorname{det} G(\widetilde{\varphi}, x))^{-\frac{1}{2}} \leqslant \operatorname{vd}(x, \Omega(\widetilde{\varphi}, x, p)) \leqslant\left(4 q^{2} p\right)^{n}(\operatorname{det} G(\widetilde{\varphi}, x))^{-\frac{1}{2}}
$$


Proof. It was shown in [1, Lm. 7] that if $C$ is a convex set containing the origin and $H(x)$ is a support function of this set, then

By Lemma 2.1.

$$
\frac{1}{\operatorname{vd}(0, C)} \leqslant \int e^{-H(x)} d x \leqslant \frac{(2 n)^{n}}{\operatorname{vd}(0, C)}
$$

$$
E\left(\widetilde{\varphi}, x, \frac{p}{q}\right) \subset \Omega(\widetilde{\varphi}, x, p) \subset E\left(\widetilde{\varphi}, x, 4 p q^{2}\right) .
$$

Without loss of generality we suppose that $x=0$. Let $H(y)$ be a support function of the domain $\Omega=\Omega(\widetilde{\varphi}, 0, p), H_{-}, H_{+}$be the support function of respectively the ellipsoids $E_{-}=E\left(\widetilde{\varphi}, 0, \frac{p}{q}\right)$ and $E_{+}=E\left(\widetilde{\varphi}, 0,4 p q^{2}\right)$. By two latter relations we have

$$
(2 n)^{-n} \operatorname{vd}\left(0, E_{-}\right) \leqslant \operatorname{vd}(0, \Omega) \leqslant \operatorname{vd}\left(0, E_{+}\right) .
$$

As it has been mentioned above, $\operatorname{vd}\left(0, E\left(\widetilde{\varphi}, 0, \frac{p}{q}\right)\right)$ is equal to the product of the principal axes, that is, $\left(\frac{p}{q}\right)^{n}\left(\lambda_{1} \ldots \lambda_{n}\right)^{-\frac{1}{2}}$, where $\lambda_{1}, \ldots, \lambda_{n}$ are the eigenvalues of the Hessian matrix $G(\widetilde{\varphi}, x)$ and at that,

$$
\operatorname{det} G(\widetilde{\varphi}, x)=\lambda_{1} \ldots \lambda_{n}
$$

The proof is complete.

Theorem 2.1. Let $\varphi \in C^{2}$ be a strictly convex function and $|\varphi(t)| \rightarrow+\infty$ as $\operatorname{dist}(t) \rightarrow 0$. If $\widetilde{\varphi}$ satisfies condition (2.1) at a point $x \in \mathbb{R}^{n}$ with $p=1$, then

$$
\frac{\left(4 q^{2}\right)^{-n}}{e(1+n !)} \sqrt{\operatorname{det} G(\widetilde{\varphi}, x)} e^{2 \widetilde{\varphi}(x)} \leqslant K(\lambda) \leqslant e^{2}\left(4 n^{2} q\right)^{n}(1+n !) \sqrt{\operatorname{det} G(\widetilde{\varphi}, x)} e^{2 \widetilde{\varphi}(x)}
$$

for $\lambda \in \mathbb{C}^{n}, x=\operatorname{Re} \lambda$.

Proof. By the Cauchy inequality for $F=\widehat{f} \in \widehat{L}_{2}(D, \varphi)$ we have:

$$
\left|\delta_{\lambda}(F)\right|^{2}=\left|\int_{D} e^{\lambda t-2 \varphi(t)} \bar{f}(t) d t\right|^{2} \leqslant\|f\|^{2} \int_{D} e^{2 x t-2 \varphi(t)} d t, \quad x=\operatorname{Re} \lambda,
$$

and this inequality becomes the identity at the function $\mathcal{E}_{\lambda}(t)=e^{\lambda t}$. Thus,

$$
K(\lambda)=\int_{D} e^{2 \operatorname{Re} \lambda t-2 \varphi(t)} d t, \quad \lambda \in \mathbb{C}^{n} .
$$

It was shown in [1, Thm. 2] that

$$
\frac{1}{e(1+n !) \operatorname{vd}(\Omega(\widetilde{\varphi}, x, 1))} e^{2 \widetilde{\varphi}(x)} \leqslant \int_{D} e^{2 x t-2 \varphi(t)} d t \leqslant \frac{e^{2}(1+n !)(2 n)^{n}}{\operatorname{vd}(\Omega(\widetilde{\varphi}, x, 1))} e^{2 \widetilde{\varphi}(x)}, \quad x \in \mathbb{R}^{n} .
$$

It remains to employ Lemma 2.2 to complete the proof.

Lemma 2.3. Let $\varphi \in C^{2}$ be a strictly convex function in a convex domain and $|\varphi(t)| \rightarrow+\infty$ as $\operatorname{dist}(t) \rightarrow 0$. Then Young adjoint function $\widetilde{\varphi}$ satisfies the Lipschitz condition:

$$
|\widetilde{\varphi}(x)-\widetilde{\varphi}(y)| \leqslant \sup _{t \in D}|t| \cdot|x-y|, \quad x, y \in \mathbb{R}^{n} .
$$

If $\widetilde{\varphi}$ satisfies condition (2.1) at the point $x \in \mathbb{R}^{n}$ with $p=1$, then

$$
\operatorname{det} G(\widetilde{\varphi}, x) \leqslant\left(16 q^{2} d^{2}\right)^{n}, \quad x \in \mathbb{R}^{n},
$$

where $d=\sup _{t \in D}|t|$. 
Proof. Let

$$
\widetilde{\varphi}(x)=x t_{x}-\varphi\left(t_{x}\right)
$$

then

$$
\widetilde{\varphi}(x)-\widetilde{\varphi}(y) \leqslant x t_{x}-\varphi\left(t_{x}\right)-\left(y t_{x}-\varphi\left(t_{x}\right)\right)=(x-y) t_{x} \leqslant d|x-y| .
$$

Swapping $x$ and $y$, we arrive at the first statement of the lemma.

It follows from the Lipschitz property that for each $x \in \mathbb{R}^{n}$ the set $\Omega(\widetilde{\varphi}, x, 1)$ contains a ball of radius $\frac{1}{2 d}$ centered at $x$. Indeed, if $|x-y| \leqslant \frac{1}{2 d}$, then since $\nabla \widetilde{\varphi}(x) \in D$ we have

$$
\widetilde{\varphi}(y)-\varphi(x)-\nabla \widetilde{\varphi}(x)(y-x) \leqslant 2 d|x-y| \leqslant 1 \text {. }
$$

Therefore,

$$
|\Omega(\widetilde{\varphi}, x, 1)| \geqslant c_{n}(2 d)^{-n}, \quad x \in \mathbb{R}^{n} .
$$

By Lemma 2.1 this implies the second statement. The proof is complete.

We take an arbitrary $\varepsilon>0$ and we let

$$
p(x, \varepsilon)=\max \left(1,\left(\operatorname{det} G(\widetilde{\varphi}, x)^{-\varepsilon}\right) .\right.
$$

Theorem 2.2. Let $\varphi \in C^{2}$ be a strictly convex function in a bounded domain $D,|\varphi(t)| \rightarrow$ $+\infty$ as $\operatorname{dist}(t) \rightarrow 0$ and $\widetilde{\varphi}$ satisfies condition (2.1) at each point $x \in \mathbb{R}^{n}$ with some $q$ independent of $x$ and $p=p(x, \varepsilon)$. Moreover, a condition holds:

$$
\frac{1}{q_{1}} \leqslant \frac{\operatorname{det} G(\widetilde{\varphi}, y)}{\operatorname{det} G(\widetilde{\varphi}, x)} \leqslant q_{1} \quad \text { as } \quad y \in E(\widetilde{\varphi}, x, p(x)), \quad x \in \mathbb{R}^{n} .
$$

for some $q_{1}>1$. Then

$$
\int_{\mathbb{R}^{n}} \frac{e^{2 y t}}{K(y)} \operatorname{det} G(\widetilde{\varphi}, y) d y \asymp e^{2 \varphi(t)}, \quad t \in D .
$$

Proof. By Theorem 2.1.

$$
\int_{\mathbb{R}^{n}} \frac{e^{2 y t}}{K(y)} \operatorname{det} G(\widetilde{\varphi}, y) d y \asymp \int_{\mathbb{R}^{n}} e^{2 y t-2 \widetilde{\varphi}(y)} \sqrt{\operatorname{det} G(\widetilde{\varphi}, y)} d y, \quad t \in D,
$$

and hence, for $x=\nabla \varphi(t)$,

$$
\int_{\mathbb{R}^{n}} \frac{e^{2 y t}}{K(y)} \operatorname{det} G(\widetilde{\varphi}, y) d y \succ \int_{E(\widetilde{\varphi}, x, 1)} e^{2 y t-2 \widetilde{\varphi}(y)} \sqrt{\operatorname{det} G(\widetilde{\varphi}, y)} d y, \quad t=\nabla \widetilde{\varphi}(x) \in D,
$$

and by condition 2.3 we get

$$
\int_{\mathbb{R}^{n}} \frac{e^{2 y t}}{K(y)} \operatorname{det} G(\widetilde{\varphi}, y) d y \succ \sqrt{\operatorname{det} G(\widetilde{\varphi}, x)} \int_{E(\widetilde{\varphi}, x, 1)} e^{2 y t-2 \widetilde{\varphi}(y)} d y, \quad t=\nabla \widetilde{\varphi}(x) \in D .
$$

Since

$$
y t-\widetilde{\varphi}(y)-\varphi(t)=-(\widetilde{\varphi}(y)-\widetilde{\varphi}(x)-\nabla \widetilde{\varphi}(x)(y-x)) \geqslant-1, \quad y \in \Omega(\widetilde{\varphi}, x, 1),
$$

and due to 2.2 the same is true for $y \in E\left(\widetilde{\varphi}, x, \frac{1}{q}\right)$. Then

$$
\int_{\mathbb{R}^{n}} \frac{e^{2 y t-2 \varphi(t)}}{K(y)} \operatorname{det} G(\widetilde{\varphi}, y) d y \succ \sqrt{\operatorname{det} G(\widetilde{\varphi}, x)}\left|E\left(\widetilde{\varphi}, x, \frac{1}{q}\right)\right|, \quad t=\nabla \widetilde{\varphi}(x) \in D,
$$


and by Lemma 2.1 ,

$$
\int_{\mathbb{R}^{n}} \frac{e^{2 y t-2 \varphi(t)}}{K(y)} \operatorname{det} G(\widetilde{\varphi}, y) d y \succ 1, \quad t \in D .
$$

We proceed to upper bounds. We let $x=\nabla \varphi(t)$ and $E(\widetilde{\varphi}, x, p(x))=E(x)$ and let us estimate the integral over the set $E(x)$. By Theorem 2.1 and by Condition (2.3),

$$
\int_{E(x)} \frac{e^{2 y t}}{K(y)} \operatorname{det} G(\widetilde{\varphi}, y) d y \prec \sqrt{\operatorname{det} G(\widetilde{\varphi}, x)} \int_{E(x)} e^{2 y t-2 \widetilde{\varphi}(y)} d y, \quad t \in D .
$$

The representation in 2.4 and Condition 2.1 imply

$$
\int_{E(x)} e^{2 y t-2 \widetilde{\varphi}(y)-2 \varphi(t)} d y \prec \int_{E(x)} e^{-\frac{1}{2 q}(y-x) G(\widetilde{\varphi}, x)(y-x)} d y \prec \int_{\mathbb{R}^{n}} e^{-\frac{1}{2 q}(y-x) G(\widetilde{\varphi}, x)(y-x)} d y .
$$

A positive definite form $G$ can be reduced to the diagonal form by means of the rotations in the space. After appropriate changes we get:

$$
\int_{E(x)} e^{2 y t-2 \widetilde{\varphi}(y)-2 \varphi(t)} d y \prec \frac{(2 q)^{\frac{n}{2}}}{\sqrt{\operatorname{det} G(\widetilde{\varphi}, x)}} \int_{\mathbb{R}^{n}} e^{-|t|^{2}} d t .
$$

By 2.5 this yields the estimate

$$
\int_{E(x)} \frac{e^{2 y t-2 \varphi(t)}}{K(y)} \operatorname{det} G(\widetilde{\varphi}, y) d y \prec 1, \quad t \in D .
$$

In order to estimate the integral over $\mathbb{R}^{n} \backslash E(x)$ we employ the boundedness of $\operatorname{det} G(\widetilde{\varphi}, x)$ proved in Lemma 2.3 and by Theorem 2.1.

$$
\begin{aligned}
\int_{\mathbb{R}^{n} \backslash E(x)} \frac{e^{2 y t-2 \varphi(t)}}{K(y)} \operatorname{det} G(\widetilde{\varphi}, y) d y & \prec \int_{\mathbb{R}^{n} \backslash E(x)} e^{2(y t-\widetilde{\varphi}(y)-\varphi(t))} \sqrt{\operatorname{det} G(\widetilde{\varphi}, y)} d y \\
& \prec \int_{\mathbb{R}^{n} \backslash E(x)} e^{2(y t-\widetilde{\varphi}(y)-\varphi(t))} d y, \quad t \in D .
\end{aligned}
$$

Let $y \in \partial E(x)$, then by condition (2.1) we have

$$
\widetilde{\varphi}(y)-\varphi(t)-x t=\widetilde{\varphi}(y)-\widetilde{\varphi}(x)-\nabla \widetilde{\varphi}(x)(y-x) \geqslant \frac{1}{2 q}(y-x) G(\widetilde{\varphi}, x)(y-x)=\frac{p}{2 q} .
$$

Hence,

$$
\widetilde{\varphi}(y)-\varphi(t)-x t \geqslant \frac{p}{2 q}, \quad y \notin E(x),
$$

and thus, $\mathbb{R}^{n} \backslash E(x) \subset \mathbb{R}^{n} \backslash \Omega\left(\widetilde{\varphi}, x, \frac{p}{2 q}\right)$. Therefore, it follows from 2.7 that

$$
\int_{\mathbb{R}^{n} \backslash E(x)} \frac{e^{2 y t-2 \varphi(t)}}{K(y)} \operatorname{det} G(\widetilde{\varphi}, y) d y \prec \int_{\mathbb{R}^{n} \backslash \Omega\left(\widetilde{\varphi}, x, \frac{p}{2 q}\right)} e^{2(y t-\widetilde{\varphi}(y)-\varphi(t))} d y, \quad t \in D .
$$

By the representation

$$
\int_{\mathbb{R}^{n} \backslash \Omega\left(\widetilde{\varphi}, x, \frac{p}{2 q}\right)} e^{2(y t-\widetilde{\varphi}(y)-\varphi(t))} d y=\int_{\frac{p(x)}{2 q}}^{\infty} e^{-2 t} d \alpha(t)
$$


we get

$$
\int_{\mathbb{R}^{n} \backslash \Omega\left(\widetilde{\varphi}, x, \frac{p}{2 q}\right)} e^{2(y t-\widetilde{\varphi}(y)-\varphi(t))} d y=\alpha\left(\frac{p(x)}{2 q}\right) e^{-\frac{p(x)}{q}}+2 \int_{\frac{p(x)}{2 q}}^{\infty} \alpha(t) e^{-2 t} d p .
$$

By Lemma 2.1.

$$
\begin{aligned}
\alpha\left(\frac{p(x)}{2 q}\right) e^{-\frac{p(x)}{q}} & \leqslant(2 q)^{n} \frac{c_{n}}{\sqrt{\operatorname{det} G(\widetilde{\varphi}, x)}}(p(x))^{\frac{n}{2}} e^{-\frac{p(x)}{q}} \\
& \leqslant(2 q)^{n} \sup _{p} p^{\frac{n}{2}+\frac{1}{\varepsilon}} e^{-\frac{p}{q}}:=(2 q)^{n} \cdot M .
\end{aligned}
$$

Owing to Minkowski inequality for mixed volumes, the function $(\alpha(t))^{\frac{1}{n}}$ is concave on $\mathbb{R}_{+}$and this is why

or

$$
(\alpha(t))^{\frac{1}{n}} \leqslant\left(\alpha\left(\frac{p(x)}{2 q}\right)\right)^{\frac{1}{n}} \frac{2 q}{p(x)} t
$$

$$
\alpha(t) \leqslant \alpha\left(\frac{p(x)}{2 q}\right)\left(\frac{2 q}{p(x)}\right)^{n} t^{n}
$$

By Lemma 2.1 and by the definition of $p(x)(p(x) \geqslant 1)$

$$
\alpha(t) \leqslant(2 q)^{2 n}(p(x))^{-\frac{n}{2}+\frac{1}{\varepsilon}} t^{n} \leqslant(2 q)^{2 n} t^{n} \quad \text { as } \quad-\frac{n}{2}+\frac{1}{\varepsilon} \leqslant 0 .
$$

If $-\frac{n}{2}+\frac{1}{\varepsilon}>0$, then for $t \geqslant \frac{p(x)}{2 q}$ we have

$$
\alpha(t) \leqslant(2 q)^{2 n}(p(x))^{-\frac{n}{2}+\frac{1}{\varepsilon}} t^{n} \leqslant(2 q)^{\frac{3 n}{2}+\frac{1}{\varepsilon}} t^{\frac{n}{2}+\frac{1}{\varepsilon}} .
$$

Hence, in each case,

$$
2 \int_{\frac{p(x)}{2 q}}^{\infty} \alpha(t) e^{-2 t} d p \leqslant M_{1}(q, \varepsilon)
$$

By 2.8 -2.10 this implies

$$
\int_{\mathbb{R}^{n} \backslash E(x)} \frac{e^{2 y t-2 \varphi(t)}}{K(y)} \operatorname{det} G(\widetilde{\varphi}, y) d y \prec 1 .
$$

In view of (2.5), (2.6) we then get the needed upper bound. The proof is complete.

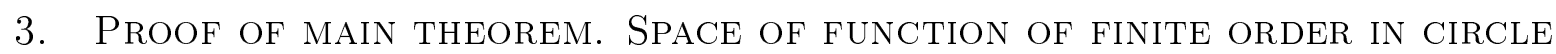

In this section we are going to prove the main result of the paper.

Theorem 3.1. Let $\varphi \in C^{2}$ be a strictly convex function in a bounded domain $D,|\varphi(t)| \rightarrow$ $+\infty$ as $\operatorname{dist}(t) \rightarrow 0$ and $\widetilde{\varphi}$ satisfies condition (2.1) at each point $x \in \mathbb{R}^{n}$ with $p=p(x)$ as well as condition 2.3$)$. Then in the space $\widehat{L}_{2}(D, \varphi)$ the norm

$$
\|F\|^{2}=\iint_{\mathbb{R}^{n}} \int_{\mathbb{R}^{n}}|F(x+i y)|^{2} \frac{\operatorname{det} G(\widetilde{\varphi}, x) d x d y}{K(x)}
$$

is equivalent to the original one induced by $L_{2}^{*}(D, \varphi)$. 
Proof. We take a function $F \in \widehat{L}_{2}(D, \varphi)$, that is, for some $f \in L_{2}(D, \varphi)$,

$$
F(x+i y)=\widehat{f}(x+i y)=\int_{D} e^{i y t}\left(e^{x t-2 \varphi(t)} \bar{f}(t)\right) d t .
$$

For a fixed $x \in \mathbb{R}^{n}$ we let

$$
g(t)=e^{x t-2 \varphi(t)} \bar{f}(t), \quad t \in D,
$$

and $g(t) \equiv 0$ as $t \notin D$. Let $\widetilde{g}$ be the classical Fourier transform of the function $g$. Then

$$
F(x+i y)=\widetilde{g}(-y), \quad y \in \mathbb{R}^{n},
$$

and by the Parseval formula

$$
\int_{\mathbb{R}^{n}}|F(x+i y)|^{2} d y=\int_{D} e^{2 x t-4 \varphi(t)}|\bar{f}(t)|^{2} d t .
$$

Therefore,

$\|F\|^{2}=\int_{\mathbb{R}^{n}}\left(\int_{\mathbb{R}^{n}}|F(x+i y)|^{2} d y\right) \frac{\operatorname{det} G(\widetilde{\varphi}, x)}{K(x)} d x=\int_{D}|\bar{f}(t)|^{2} e^{-4 \varphi(t)}\left(\int_{\mathbb{R}^{n}} \frac{e^{2 x t}}{K(x)} \operatorname{det} G(\widetilde{\varphi}, x) d x\right) d t$.

By Theorem 2.2,

$$
\|F\|^{2} \asymp \int_{D}|\bar{f}(t)|^{2} e^{-2 \varphi(t)} d t
$$

The proof is complete.

Remark 3.1. Since statement of Theorem 3.1 is of an asymptotic nature, then the following theorem holds true as well.

Theorem 3.2. Let $\varphi \in C^{2}$ be a convex function in a bounded domain $D$ and is strictly convex in the vicinity of the boundary of $D,|\nabla \varphi(t)| \rightarrow+\infty$ as $\operatorname{dist}(t) \rightarrow 0$ and $\widetilde{\varphi}$ satisfies condition (2.1) at the points $x \in \mathbb{R}^{n}$ with a sufficiently large absolute value with $p=p(x)$ as well as condition (2.3). Then in the space $\widehat{L}_{2}(D, \varphi)$ the norm

$$
\|F\|^{2}=\int_{\mathbb{R}^{n}} \int_{\mathbb{R}^{n}}|F(x+i y)|^{2} \frac{\operatorname{det} G(\widetilde{\varphi}, x) d x d y}{K(x)}
$$

is equivalent to the original one induced by $L_{2}^{*}(D, \varphi)$.

As an example we consider the functions $\varphi(t)=a(1-|t|)^{-\beta}, \beta<0$, in the unit circle $B(0,1)$. By straightforward calculations we find:

$$
\widetilde{\varphi}(x)=|x|-c|x|^{\alpha}, \quad x \in \mathbb{R}^{n},
$$

where $\alpha=\frac{\beta}{\beta+1}$ and $c=(a \beta)^{\frac{1}{\beta+1}}(a+1)$. For the sake of simplicity we suppose that $c=1$ and $n=2$ :

$$
\widetilde{\varphi}(x)=|x|-|x|^{\alpha}, \quad x \in \mathbb{R}^{2} .
$$

Let us confirm that the assumptions of Theorem 3.1 are satisfied. By the radial property we consider the points at the ray $x=(t, 0), t>0$. By straightforward calculations we find the gradient vector

$$
\nabla \widetilde{\varphi}(x)=\left(x_{1}\left(|x|^{-1}-\alpha|x|^{\alpha-2}\right), \quad x_{2}\left(|x|^{-1}-\alpha|x|^{\alpha-2}\right)\right),
$$


and the Hessian matrix

$$
\left\{\begin{array}{l}
\frac{\partial^{2} \widetilde{\varphi}(x)}{\partial x_{1}^{2}}=x_{2}^{2}|x|^{-3}-\alpha|x|^{\alpha-2}-\alpha(\alpha-2)|x|^{\alpha-4} x_{1}^{2} \\
\frac{\partial^{2} \widetilde{\varphi}(x)}{\partial x_{2}^{2}}=x_{1}^{2}|x|^{-3}-\alpha|x|^{\alpha-2}-\alpha(\alpha-2)|x|^{\alpha-4} x_{2}^{2}, \\
\frac{\partial^{2} \widetilde{\varphi}(x)}{\partial x_{1} \partial x_{2}}=-x_{1} x_{2}\left(|x|^{-3}+\alpha(\alpha-2)|x|^{\alpha-4}\right) .
\end{array}\right.
$$

At the point $x_{0}=(t, 0)$ we have:

$$
\begin{aligned}
& \nabla \widetilde{\varphi}\left(x_{0}\right)=\left(1-\alpha t^{\alpha-1}, 0\right), \\
& \left\{\begin{array}{l}
\frac{\partial^{2} \widetilde{\varphi}\left(x_{0}\right)}{\partial x_{1}^{2}}=\alpha(1-\alpha) t^{\alpha-2}, \\
\frac{\partial^{2} \widetilde{\varphi}\left(x_{0}\right)}{\partial x_{2}^{2}}=t^{-1}-\alpha t^{\alpha-2}, \\
\frac{\partial^{2} \widetilde{\varphi}\left(x_{0}\right)}{\partial x_{1} \partial x_{2}}=0 .
\end{array}\right.
\end{aligned}
$$

At the point $x_{0}$ the Hessian matrix is of the diagonal form and therefore,

$$
\lambda_{1}\left(x_{0}\right)=\alpha(1-\alpha) t^{\alpha-2}, \quad \lambda_{2}\left(x_{0}\right)=t^{-1}+(1-\alpha) \alpha t^{\alpha-2}
$$

are the eigenvalues of the matrix $G\left(\widetilde{\varphi}, x_{0}\right)$ and for $\varepsilon=\frac{\alpha}{2(3-\alpha)}$ we get

$$
p\left(x_{0}\right) \asymp t^{\frac{\alpha}{2}}, \quad t \rightarrow \infty .
$$

We proceed to checking conditions (2.1) and (2.3). We first estimate the principal axes of the ellipse $E\left(\widetilde{\varphi}, x_{0}, p\left(x_{0}\right)\right)$ :

$$
a_{1}\left(x_{0}\right)=\sqrt{\frac{p\left(x_{0}\right)}{\lambda_{1}\left(x_{0}\right)}} \asymp t^{1-\frac{\alpha}{4}}, \quad a_{2}\left(x_{0}\right)=\sqrt{\frac{p\left(x_{0}\right)}{\lambda_{2}\left(x_{0}\right)}} \asymp t^{\frac{1}{2}+\frac{\alpha}{4}},
$$

in particular, for $x \in E\left(\widetilde{\varphi}, x_{0}, p\left(x_{0}\right)\right)$,

$$
\left|x_{2}\right| \prec t^{\frac{1}{2}+\frac{\alpha}{4}}, \quad\left|x_{1}\right| \asymp\left|x_{0}\right|=t, \quad\left|x-x_{0}\right| \asymp t .
$$

Let $x \in E\left(\widetilde{\varphi}, x_{0}, p\left(x_{0}\right)\right)$ and $\omega=\frac{x-x_{0}}{\left|x-x_{0}\right|}, x=y \omega+x_{0}, u(y)=\widetilde{\varphi}\left(y \omega+x_{0}\right), y>0$. By (3.3) we obtain:

$$
\frac{\partial^{2} \widetilde{\varphi}(x)}{\partial x_{1}^{2}} \omega_{1}^{2} \prec t^{\alpha-2} \omega_{1}^{2}, \quad \frac{\partial^{2} \widetilde{\varphi}(x)}{\partial x_{2}^{2}} \omega_{2}^{2} \prec t^{-1} \omega_{2}^{2} .
$$

Since $x_{2}=\left|x-x_{0}\right| \omega_{2}$, then

$$
\left|\frac{\partial^{2} \widetilde{\varphi}(x)}{\partial x_{1} \partial x_{2}} \omega_{1} \omega_{2}\right| \prec t^{-1} \omega_{2}^{2} .
$$

By (3.2), (3.4) this implies that

$$
\omega G(\widetilde{\varphi}, x) \omega \prec \omega G\left(\widetilde{\varphi}, x_{0}\right) \omega .
$$

Swapping $x_{0}$ and $x$, we get

$$
\omega G(\widetilde{\varphi}, x) \omega \asymp \omega G\left(\widetilde{\varphi}, x_{0}\right) \omega .
$$

By the mean value theorem,

$$
\widetilde{\varphi}(x)-\widetilde{\varphi}\left(x_{0}\right)-\nabla \widetilde{\varphi}\left(x_{0}\right)\left(x-x_{0}\right)=\left(x-x_{0}\right) G\left(\widetilde{\varphi}, x^{*}\right)\left(x-x_{0}\right),
$$

where $x^{*}$ is a point in the segment connecting $x_{0}$ with $x$. Relation (3.5) implies:

$$
\widetilde{\varphi}(x)-\widetilde{\varphi}\left(x_{0}\right)-\nabla \widetilde{\varphi}\left(x_{0}\right)\left(x-x_{0}\right) \asymp\left(x-x_{0}\right) G\left(\widetilde{\varphi}, x^{*}\right)\left(x-x_{0}\right),
$$


that is, condition (2.1) is satisfied.

By $(3.2) \operatorname{det} G(\widetilde{\varphi}, x) \asymp|x|^{\alpha-3}$ and this is why condition (2.3) obviously holds. Thus, the following theorem holds true.

Theorem 3.3. If $D=\left\{t \in \mathbb{R}^{2},|t|<1, \varphi(t)=a(1-|t|)^{-\beta}, \beta<0\right\}$, then the space $\widehat{L}_{2}(D, \varphi)$ regarded as a normed space is isomoprhic to the space of entire functions $F(z), z=x+i y \in \mathbb{C}^{2}$ with

$$
\|F\|^{2}:=\int|F(x+i y)|^{2} e^{-2|x|-2(a \beta)^{\frac{1}{\beta+1}}(a+1)|x|^{\frac{\beta}{\beta+1}}}(1+|x|)^{\frac{\alpha-3}{2}} d x d y<\infty,
$$

where $\alpha=\frac{\beta}{\beta+1}$.

\section{BIBLIOGRAPHY}

1. R.A. Bashmakov, K.P. Isaev, R.S. Yulmukhametov. On geometric characteristics of convex functions and Laplace integrals // Ufimskij Matem. Zhurn. 2:1, 3-16 (2010).

2. V.I. Lutsenko, R.S. Yulmukhametov. Generalization of the Paley-Wiener theorem in weighted spaces // Matem. Zamet. 48:5, 80-87 (1990). [Math. Notes. 48:5, 1131-1136 (1990).]

Rinad Salavatovich Yulmukhametov,

Institute of Mathematics,

Ufa Federal Research Center, RAS,

Chernyshevsky str. 112,

450008, Ufa, Russia

E-mail: Yulmukhametov@mail.ru 